\title{
Cubic Spline Prewavelets on the Four-Directional Mesh
}

\author{
M. D. Buhmann'1), O. Davydov²) and T. N. T. Goodman ${ }^{3)}$
}

\begin{abstract}
In this paper, we design differentiable, twodimensional, piecewise polynomial cubic prewavelets of particularly small compact support. They are given in closed form, and provide stable, orthogonal decompositions of $L^{2}\left(\mathbb{R}^{2}\right)$. In particular, the splines we use in our prewavelet constructions give rise to stable bases of spline spaces that contain all cubic polynomials, whereas the more familiar box spline constructions cannot reproduce all cubic polynomials, unless resorting to a box spline of higher polynomial degree.
\end{abstract}

Dedicated to Professor M.J.D. Powell on the occasion of his 65th birthday and his retirement.

\section{$\S 1$. Introduction}

At the present time, there is a particularly attractive research area in approximation theory, namely the theory and practice of wavelet decomposition of signals and functions. They are highly relevant to the foundations of numerical analysis, as one of the basic instruments for state-of-the-art numerical methods $[9,16,13]$, for example.

Their applications include the numerical solution of partial differential equations, especially when Galerkin approaches are used $[9,16]$. In this application, bivariate splines, that is finite elements in the language of PDE solvers, are important as well as particular properties of the generating functions, namely small, compact support. In this note we propose spline prewavelets of small support that can be useful for such practical use. The small support is closely related to the sparsity in the stiffness or mass matrices which come up in PDE applications.

We remark already at this point that there is a host of construction of univariate prewavelets and wavelets which may be generalised in a simple way by tensor production methods to multiple dimensions. We avoid this approach because it usually leads to much larger support sizes than required (in some highly complicated situations, however, such as wavelets on irregular bounded domains, local tensor product constructions of continuous wavelets are very suitable, see, e.g. [9]). After all we are aiming at small

1) Mathematical Institute, Justus Liebig University, D-35392 Giessen, Germany,

martin.buhmann@math.uni-giessen.de

2) Mathematical Institute, Justus Liebig University, D-35392 Giessen, Germany, oleg.davydov@math.uni-giessen.de

3) Department of Mathematics, The University of Dundee, Dundee DD1 4HN, U.K. tgoodman@mcs.dundee.ac.uk 
supports in this work. This is true both for spline constructions and for example for Daubechies wavelets [13]; in this paper we focus on spline (pre)wavelets.

Apart from the aforementioned univariate constructions, there are several multivariate spline constructions. Especially for continuous wavelets on non-uniform meshes, there are many articles for one and more dimensional constructions $[3,12,17,27]$. There are also very general approaches to prewavelets in [1], for instance, or see the related paper [24], while we wish to get very explicit constructions in this article. Nonetheless, our construction is based on the basic ideas for generating prewavelets as in [1].

Several other quite explicit constructions in the literature use $C^{1}$ hierarchical bases $[11,15,25]$, and there are other constructions of splines and hierarchical bases either on the four-directional mesh $[7,8,19]$ or on the so-called Fraeijs de Veubeke-Sander (FVS) triangulations $[5,18,20,26]$. By contrast, continuously differentiable spline-based prewavelets on $\mathbb{R}^{d}$ for more than one dimension $(d \geq 2)$ are available only as tensor product or box spline constructions. The box spline constructions suffer from the problem that the stable constructions generate a spline space which does not contain all polynomials of corresponding degree in two dimensions (see, e.g., [2]) and that box spline on the fourdirectional mesh are no longer stable (see, e.g., [10], [7]). By contrast, our subsequent construction is based on stable piecewise cubic bases which generate all cubic bivariate polynomials. Therefore, they give prewavelets orthogonal to all cubic polynomials and possess so-called vanishing moments [12] of component degree at most three. Also, they are on the four-directional mesh which provides more symmetry than the threedirectional mesh which has an undesirable bias. This is the reason why, for instance, the famous Zwart-Powell element $[22,28]$ that is a quadratic piecewise polynomial on the four-directional mesh is so popular. To remind the reader of the concepts of three and four-directional meshes, we recall that the latter is the triangulation of $\mathbb{R}^{2}$ generated by the four families of parallel lines $x_{1}=k, x_{2}=k, x_{1}-x_{2}=k, x_{1}+x_{2}=k, k \in \mathbb{Z}[6]$, while the former comes from leaving out the $x_{1}+x_{2}=k$ lines in the construction.

In order to introduce prewavelets formally in this article, we have to recall the definition of a multiresolution analysis. This is always an infinite nested sequence of closed subspaces $V_{j} \subset L^{2}\left(\mathbb{R}^{d}\right), j \in \mathbb{Z}$,

$$
\{0\} \subset \cdots \subset V_{-1} \subset V_{0} \subset V_{1} \subset \cdots \subset L^{2}\left(\mathbb{R}^{d}\right)
$$

that satisfy the following three fundamental properties:

(i) $f \in V_{j} \Leftrightarrow f(2 \cdot) \in V_{j+1}$ for all integers $j$,

(ii)

$$
\bigcap_{j=-\infty}^{\infty} V_{j}=\{0\}, \quad \overline{\bigcup_{j=-\infty}^{\infty} V_{j}}=L^{2}\left(\mathbb{R}^{d}\right),
$$

(but see [1] for conditions under which (ii) is redundant), (iii) there is a Riesz basis $\left\{\varphi_{i}: i \in I\right\}$ of $V_{0}$, i.e.,

$$
V_{0}=\operatorname{span}_{\ell^{2}(I)}\left\{\varphi_{i}: i \in I\right\}
$$


where $I$ is a countable index set, the coefficients of the spanning functions are always square-summable as indicated by the subscript, and there exist positive and finite constants $K_{1}>0$ and $K_{2}<\infty$ such that for all $c \in \ell^{2}(I)$

$$
K_{1}\|c\|_{2} \leq\left\|\sum_{i \in I} c_{i} \varphi_{i}\right\|_{2} \leq K_{2}\|c\|_{2} .
$$

Here we use the notation $c=\left(c_{i}\right)_{i \in I}$; the 2-norms conveniently denote the Euclidean norm on $\ell^{2}(I)$ or on $L^{2}\left(\mathbb{R}^{d}\right)$ as is appropriate from the context.

The above Riesz basis property is of particular importance with respect to the stability of the computations of the coefficients of an expansion. Unless this property is provided, instabilities can occur through cancellations of coefficients of a function's expansion in the infinite basis.

It is usually advantageous to use so-called shift-invariant spaces $V_{j}$ so long as this is possible; the spaces generated in the above MRA are usually called shift-invariant as soon as, if an element $f$ is contained in them, then any shift by an integer times $2^{-j}$, depending on the index of $V_{j}$, is as well. If the space originates from just one function that is translated, i.e., for instance, $V_{0}$ is spanned by $\varphi$ and its multiinteger shifts, then it is called a principal shift-invariant space (PSI). If several (finitely many) functions are used and translated to span the space, then the latter is called a finitely generated (FSI) shift-invariant space. de Boor, DeVore and Ron have analysed PSI and FSI spaces in a series of papers, not only in the wavelet or prewavelet context, of which [1] is just one. Throughout this paper, we shall be dealing with finitely generated shift-invariant spaces. (See, however, Remark 4.5.) To begin with, our construction relies on a very explicit computation of the generators of the FSI spaces $V_{0}$ and $V_{1}$ which will be the theme of the next section.

Before we begin with this, however, we observe that the properties (i)-(iii) of multiresolution analysis have many fundamental consequences. One of them is that we can find a collection of square-integrable functions named prewavelets in $V_{1} \backslash\{0\}$, which are orthogonal to $V_{0}$, call the set of prewavelets $\Psi$, whose translates span a space $W=V_{1} \ominus V_{0}$. In other words, $V_{1}$ is the direct and orthogonal sum of $V_{0}$ and the space $W$, for which $W=V_{1} \ominus V_{0}$ is a short notation. In principle, all functions of $W$, and indeed the aforementioned spanning set, can be found by computing the error of a least-squares projection of all elements from $V_{1}$ onto $V_{0}$, i.e., the element of $V_{1}$ minus its projection onto $V_{0}$, and this is how we find our spanning set for $W$. Indeed, the whole construction relies on finding a suitable set of generating functions (in the event, they are differentiable cubic splines of small support) in $V_{1}$ whose projection is then computed to form the prewavelets. Given that $V_{1}$ and $V_{0}$ are FSI spaces in our context (their generators are specified later-on), $W$ will be an FSI space too.

The most important consequence of the properties of multiresolution analysis and the properties of $W$ are that we get the infinite decomposition

$$
L^{2}\left(\mathbb{R}^{d}\right)=\bigoplus_{j=-\infty}^{\infty} W_{j}
$$


Here $W_{j}$ denotes $W$ with the functions scaled by $2^{j}$, see for instance $[16,1]$ or many other standard works on wavelets or prewavelets.

In summary, we have the desired decomposition of the whole of $L^{2}\left(\mathbb{R}^{d}\right)$, because the $W_{j}$ are mutually orthogonal which follows from a standard argument using the fact that the prewavelets $\psi$ are orthogonal to $V_{0}$ and from (i). The prewavelets are called wavelets if their translates on the same scale are also mutually orthonormal. We will not perform the final orthogonalisation step in this paper here and rely on prewavelets instead, because compact support for spline prewavelets is usually lost when they are orthogonalised to become wavelets. A general construction of prewavelets from shiftinvariant spaces is to be found in [1].

The goal is to obtain an explicit construction of prewavelets spanned by a finite linear combination of splines from $V_{0}$ and $V_{1}$ with rational coefficients which enables us to get prewavelets with small support. Moreover, we will use piecewise cubic splines and construct prewavelets as opposed to wavelets. We note that not all piecewise polynomial spaces in more than one dimension are able to provide multiresolution analyses and are indeed refinable. The construction in this paper provides this property, as does, for example, the construction of splines in two dimensions with the famous Powell-Sabin split [23].

The finding of biorthogonal dual functions with especially small support is very relevant to our construction because it facilitates the computation of the aforementioned projections (see a similar approach to computing continuous prewavelets in [27, 12]), as is a general finite element basis for the four-directional mesh with which we shall begin. To start now, we have a notation for the mesh generated by the four directions and for the spline space thereon: Let $\triangle$ denote the four-directional mesh as defined in the introduction above, and let $S_{3}^{1}(\triangle)$ be the space of all square integrable $C^{1}$ cubic splines with respect to $\triangle$,

$$
S_{3}^{1}(\triangle)=\left\{s \in C^{1}\left(\mathbb{R}^{2}\right) \cap L^{2}\left(\mathbb{R}^{2}\right):\left.s\right|_{T} \in \Pi_{3}, T \in \triangle\right\},
$$

where $\Pi_{3}$ denotes the space of bivariate polynomials of total degree at most three. This is our $V_{0}$ in the notation for multiresolution analyses above. Both $V_{0}$ and $V_{1}$ will play the dominant rôles in our construction, because they are needed to find the prewavelets, while all other $V_{j}$ and $W_{j}$ are found trivially by dilation.

\section{$\S$ 2. Bases for $S_{3}^{1}(\triangle)$}

\subsection{Finite-element basis}

Since $\triangle$ is a special case of the FVS triangulation (quadrangulation with both diagonals added to each quadrilateral), we start by describing the classical finite-element basis for it 
$[18,26]$ (see also $[5,19])$. To this end, consider the set of nodal functionals on $V_{0}:=S_{3}^{1}(\triangle)$

$$
\begin{aligned}
& \sigma_{1, k} s=s(k), \quad k \in \mathbb{Z}^{2}, \\
& \sigma_{2, k} s=D_{x_{1}} s(k), \quad k \in \mathbb{Z}^{2}, \\
& \sigma_{3, k} s=D_{x_{2}} s(k), \quad k \in \mathbb{Z}^{2}, \\
& \sigma_{4, k} s=D_{x_{1}} s\left(k+\left(0, \frac{1}{2}\right)\right), \quad k \in \mathbb{Z}^{2}, \\
& \sigma_{5, k} s=D_{x_{2}} s\left(k+\left(\frac{1}{2}, 0\right)\right), \quad k \in \mathbb{Z}^{2},
\end{aligned}
$$

where $D_{x_{1}}, D_{x_{2}}$ denote the derivatives in $x_{1}$ and $x_{2}$, respectively. The finite element basis functions $s_{i, k} \in S_{3}^{1}(\triangle), i=1, \ldots, 5, k \in \mathbb{Z}^{2}$, are required to give the duality condition

$$
\sigma_{j, \ell} s_{i, k}= \begin{cases}1, & \text { if } j=i \text { and } \ell=k \\ 0, & \text { otherwise }\end{cases}
$$

By the well-posedness of the corresponding finite-element interpolation scheme, it follows that

$$
\operatorname{supp} s_{i, k}=\left\{\begin{array}{ll}
{[-1,1]^{2}+k,} & i=1,2,3, \\
{[-1,1] \times[0,1]+k,} & i=4, \\
{[0,1] \times[-1,1]+k,} & i=5,
\end{array} \quad k \in \mathbb{Z}^{2} .\right.
$$

The uniformity and symmetry of the triangulation imply that all basis functions are integer translates of the five functions $s_{i}:=s_{i, 0}, i=1, \ldots, 5$,

$$
s_{i, k}=s_{i}(\cdot-k), \quad i=1, \ldots, 5, \quad k \in \mathbb{Z}^{2} .
$$

Moreover, it is easy to see that

$$
s_{3}\left(x_{1}, x_{2}\right)=s_{2}\left(x_{2}, x_{1}\right), \quad s_{5}\left(x_{1}, x_{2}\right)=s_{4}\left(x_{2}, x_{1}\right),
$$

and the functions $s_{1}, s_{2}, s_{4}$ possess the following symmetries:

$$
\begin{aligned}
& s_{1}\left(x_{1}, x_{2}\right)=s_{1}\left(x_{2}, x_{1}\right)=s_{1}\left(\left|x_{1}\right|,\left|x_{2}\right|\right), \\
& s_{2}\left(x_{1}, x_{2}\right)=s_{2}\left(x_{1},-x_{2}\right)=-s_{2}\left(-x_{1}, x_{2}\right), \\
& s_{4}\left(x_{1}, x_{2}\right)=s_{4}\left(x_{1}, 1-x_{2}\right)=-s_{4}\left(-x_{1}, x_{2}\right) .
\end{aligned}
$$

Let us denote the set of all functions $s_{i, k}$ by $\mathcal{B}_{\mathrm{FE}}$,

$$
\mathcal{B}_{\mathrm{FE}}=\left\{s_{i, k}=s_{i}(\cdot-k): i=1, \ldots, 5, k \in \mathbb{Z}^{2}\right\} .
$$

By using the explicit dual basis (2.1) and the locality of the supports of $s_{i, k}(2.2)$, it is easy to show (see e.g. Lemma 6.2 in [14]) that $\mathcal{B}_{\mathrm{FE}}$ is a Riesz basis for $S_{3}^{1}(\triangle$ ), i.e., for any square summable coefficient vector $c$, it is true that

$$
K_{1}\|c\|_{\ell^{2}} \leq\left\|\sum_{i=1}^{5} \sum_{k \in \mathbb{Z}^{2}} c_{i, k} s_{i}(\cdot-k)\right\|_{L^{2}\left(\mathbb{R}^{2}\right)} \leq K_{2}\|c\|_{\ell^{2}}
$$


where $K_{1}, K_{2}>0$ are some finite absolute constants. In fact, this holds for all $L^{p}$.

Another important feature of the finite-element basis $\mathcal{B}_{\mathrm{FE}}$ is that it is locally linearly independent (LLI) with respect to the partition

$$
\mathbb{R}^{2}=\bigcup_{k \in \mathbb{Z}^{2}}\left([0,1]^{2}+k\right)
$$

i.e., for each $k \in \mathbb{Z}^{2}$, the set

$$
\left.\mathcal{B}_{\mathrm{FE}}\right|_{[0,1]^{2}+k}:=\left\{\left.s\right|_{[0,1]^{2}+k}: s \in \mathcal{B}_{\mathrm{FE}}, \operatorname{supp} s \cap\left((0,1)^{2}+k\right) \neq \emptyset\right\}
$$

is a basis for $\left.S_{3}^{1}(\triangle)\right|_{[0,1]^{2}+k}$. Indeed, it follows from the theory of FVS element that $\left.\operatorname{dim} S_{3}^{1}(\triangle)\right|_{[0,1]^{2}+k}=16$. Since $\left.\mathcal{B}_{\mathrm{FE}}\right|_{[0,1]^{2}+k}$ is a spanning set for $\left.S_{3}^{1}(\triangle)\right|_{[0,1]^{2}+k}$ and $\left.\# \mathcal{B}_{\mathrm{FE}}\right|_{[0,1]^{2}+k}=16$, local linear independence follows.

As a consequence, the basis $\mathcal{B}_{\mathrm{FE}}$ has the following support property (cf. [4]): if $s \in S_{3}^{1}(\triangle)$ and $\operatorname{supp} s \subseteq M$, where

$$
M=\bigcup_{k \in \mathcal{K}}\left([0,1]^{2}+k\right), \quad \text { for some } \quad \mathcal{K} \subseteq \mathbb{Z}^{2}
$$

then

$$
s=\sum_{\substack{i, k \\ \operatorname{supp} s_{i, k} \subseteq M}} c_{i, k} s_{i, k} .
$$

Indeed, if $k \notin \mathcal{K}$, then $\left.s\right|_{[0,1]^{2}+k} \equiv 0$ and, by LLI, all coefficients $c_{i, k}$ of $s$ such that supp $s \cap\left((0,1)^{2}+k\right) \neq \emptyset$ must be zero. The support property of the scaled version $\mathcal{B}_{\mathrm{FE}}\left(\frac{1}{2} \triangle\right)$ of $\mathcal{B}_{\mathrm{FE}}$ will be used in the proof of Theorem 3.1.

Remark 2.1. Note that $\mathcal{B}_{\mathrm{FE}}$ is not LLI with respect to $\triangle$.

In what follows we will use the Bernstein-Bézier representations of the basis splines for $S_{3}^{1}(\triangle)$ introduced in [19]. Using the notation of [19], we have the following relations: $l s^{(0,0)}=s_{1}, l s^{(1,0)}=s_{2}+\frac{1}{2} s_{4}+\frac{1}{2} s_{4,(0,-1)}, l s^{(1,2)}=-\frac{1}{8} s_{4}$. Figures 1 and 2 in [19] can be used to compute the Bernstein-Bézier coefficients of the finite element basis splines we will refer to in some calculations.

\subsection{Modified basis}

We set

$$
t_{1}:=s_{1}
$$

Using the Bernstein-Bézier representations of the functions $s_{1}, \ldots, s_{5}$, it is easy to see that the function

$$
t_{2}:=s_{2}-s_{3}-\frac{1}{4}\left(s_{4}+s_{4,(0,-1)}-s_{5}-s_{5,(-1,0)}\right)
$$


has a smaller support than $s_{2}$, namely

$$
\operatorname{supp} t_{2}=\left\{\left(x_{1}, x_{2}\right) \in \mathbb{R}^{2}:\left|x_{1}\right| \leq 1,\left|x_{2}\right| \leq 1,\left|x_{1}-x_{2}\right| \leq 1\right\} .
$$

Similarly, the function

$$
t_{3}:=-s_{2}-s_{3}+\frac{1}{4}\left(s_{4}+s_{4,(0,-1)}+s_{5}+s_{5,(-1,0)}\right)
$$

has a smaller support than $s_{3}$, viz.

$$
\operatorname{supp} t_{3}=\left\{\left(x_{1}, x_{2}\right) \in \mathbb{R}^{2}:\left|x_{1}\right| \leq 1,\left|x_{2}\right| \leq 1,\left|x_{1}+x_{2}\right| \leq 1\right\}
$$

and we have

$$
t_{3}\left(x_{1}, x_{2}\right)=t_{2}\left(-x_{1}, x_{2}\right) .
$$

Therefore, we want to construct a new Riesz basis for $S_{3}^{1}(\triangle)$ using $t_{2}, t_{3}$ as generators instead of $s_{2}, s_{3}$.

Moreover, there is a subtle technical reason (which will become clear later, see Remark 3.2) for replacing $s_{4}, s_{5}$ along with their translates $s_{4,(0,-1)}, s_{5,(-1,0)}$ by the functions

$$
\begin{aligned}
t_{4}^{+} & :=s_{4}+s_{4,(0,-1)}, \\
t_{4}^{-} & :=s_{4}-s_{4,(0,-1)}, \\
t_{5}^{+} & :=s_{5}+s_{5,(-1,0)}, \\
t_{5}^{-} & :=s_{5}-s_{5,(-1,0)}
\end{aligned}
$$

Then

$$
t_{5}^{+}\left(x_{1}, x_{2}\right)=t_{4}^{+}\left(x_{2}, x_{1}\right), \quad t_{5}^{-}\left(x_{1}, x_{2}\right)=t_{4}^{-}\left(x_{2}, x_{1}\right),
$$

and

$$
\operatorname{supp} t_{4}^{ \pm}=\operatorname{supp} t_{5}^{ \pm}=[-1,1]^{2} \text {. }
$$

It is easy to see that the functions $t_{1}, t_{2}, t_{4}^{ \pm}$possess the following symmetries:

$$
\begin{aligned}
& t_{1}\left(x_{1}, x_{2}\right)=t_{1}\left(x_{2}, x_{1}\right)=t_{1}\left(\left|x_{1}\right|,\left|x_{2}\right|\right), \\
& t_{2}\left(x_{1}, x_{2}\right)=t_{2}\left(-x_{2},-x_{1}\right)=-t_{2}\left(x_{2}, x_{1}\right), \\
& t_{4}^{+}\left(x_{1}, x_{2}\right)=t_{4}^{+}\left(x_{1},-x_{2}\right)=-t_{4}^{+}\left(-x_{1}, x_{2}\right), \\
& t_{4}^{-}\left(x_{1}, x_{2}\right)=-t_{4}^{-}\left(-x_{1}, x_{2}\right)=-t_{4}^{-}\left(x_{1},-x_{2}\right) .
\end{aligned}
$$

We set

$$
\mathcal{B}:=\left\{t_{i, k}: i=1, \ldots, 5, k \in \mathbb{Z}^{2}\right\}
$$

where

$$
\begin{aligned}
t_{i, k} & :=t_{i}(\cdot-k) \quad i=1,2,3, \quad k \in \mathbb{Z}^{2}, \\
t_{4, k} & := \begin{cases}t_{4}^{+}(\cdot-k), & \text { if } k \in \mathbb{Z} \times 2 \mathbb{Z}, \\
t_{4}^{-}(\cdot-k+(0,-1)), & \text { if } k \in \mathbb{Z} \times(2 \mathbb{Z}-1),\end{cases} \\
t_{5, k} & := \begin{cases}t_{5}^{+}(\cdot-k), & \text { if } k \in 2 \mathbb{Z} \times \mathbb{Z}, \\
t_{5}^{-}(\cdot-k+(-1,0)), & \text { if } k \in(2 \mathbb{Z}-1) \times \mathbb{Z} .\end{cases}
\end{aligned}
$$


Since

$$
\begin{aligned}
& s_{1, k}=t_{1, k}, \quad k \in \mathbb{Z}^{2}, \\
& s_{2, k}=\left(t_{2, k}-t_{3, k}\right) / 2+\frac{1}{4}\left(s_{4, k}+s_{4, k+(0,-1)}, \quad k \in \mathbb{Z}^{2},\right. \\
& s_{3, k}=-\left(t_{2, k}+t_{3, k}\right) / 2+\frac{1}{4}\left(s_{5, k}+s_{5, k+(-1,0)}\right), \quad k \in \mathbb{Z}^{2}, \\
& s_{4, k}= \begin{cases}\left(t_{4, k}+t_{4, k+(0,-1)}\right) / 2, & k \in \mathbb{Z} \times 2 \mathbb{Z}, \\
\left(t_{4, k+(0,1)}-t_{4, k}\right) / 2, & k \in \mathbb{Z} \times(2 \mathbb{Z}-1),\end{cases} \\
& s_{5, k}= \begin{cases}\left(t_{5, k}+t_{5, k+(-1,0)}\right) / 2, & k \in 2 \mathbb{Z} \times \mathbb{Z}, \\
\left(t_{5, k+(1,0)}-t_{5, k}\right) / 2, & k \in(2 \mathbb{Z}-1) \times \mathbb{Z},\end{cases}
\end{aligned}
$$

the transformation from $\mathcal{B}_{\mathrm{FE}}$ to $\mathcal{B}$ and back can be done with the help of multiplication by band matrices, which implies that $\mathcal{B}$ is also a Riesz basis for $S_{3}^{1}(\triangle)$.

It is easy to see that $\mathcal{B}$ is LLI and hence has the aforementioned support property with respect to the partition

$$
\mathbb{R}^{2}=\bigcup_{k \in \mathbb{Z}^{2}}\left([-1,1]^{2}+2 k\right)
$$

\section{§3. Biorthogonal dual functions}

Let the triangulation $\frac{1}{2} \triangle$ be the refined four-directional mesh generated by the parallel lines $x_{1}=k / 2, x_{2}=k / 2, x_{1}-x_{2}=k / 2, x_{1}+x_{2}=k / 2, k \in \mathbb{Z}$. Obviously, the space $S_{3}^{1}\left(\frac{1}{2} \triangle\right)$ of $C^{1}$ cubics with respect to $\frac{1}{2} \triangle$ contains all elements of $S_{3}^{1}(\triangle)$,

$$
V_{0}=S_{3}^{1}(\triangle) \subset S_{3}^{1}\left(\frac{1}{2} \triangle\right)=V_{1} .
$$

By scaling we obtain a finite-element Riesz basis for the space $S_{3}^{1}\left(\frac{1}{2} \triangle\right)$,

$$
\mathcal{B}_{\mathrm{FE}}\left(\frac{1}{2} \triangle\right):=\left\{s_{i, k}(2 \cdot): i=1, \ldots, 5, \quad k \in \mathbb{Z}^{2}\right\}
$$

which is LII with respect to the partition

$$
\mathbb{R}^{2}=\bigcup_{k \in \mathbb{Z}^{2}}\left([0,1 / 2]^{2}+k / 2\right),
$$

and has the support property with respect to it.

We now want to construct a set $\tilde{\mathcal{B}} \subset S_{3}^{1}\left(\frac{1}{2} \triangle\right)$ of biorthogonal dual functions (with respect to $\mathcal{B})$, that we call

$$
\tilde{\mathcal{B}}:=\left\{\tilde{t}_{i, k}: i=1, \ldots, 5, k \in \mathbb{Z}^{2}\right\}
$$

such that

$$
\left\langle\tilde{t}_{i, k}, t_{i^{\prime}, k^{\prime}}\right\rangle=\left\{\begin{array}{cl}
\left\|t_{i, k}\right\|_{2}^{2}, & \text { if } i=i^{\prime} \text { and } k=k^{\prime} \\
0, & \text { otherwise }
\end{array}\right.
$$


where

$$
\langle f, g\rangle:=\int_{\mathbf{R}^{2}} f g .
$$

However, the condition (3.1) does not determine the set $\tilde{\mathcal{B}}$ uniquely since the space $S_{3}^{1}\left(\frac{1}{2} \triangle\right)$ is much richer than $S_{3}^{1}(\triangle)$. Therefore, we assume that $\tilde{t}_{i, k}$ have the same support and symmetry properties as $t_{i, k}$ to take up the extra degrees of freedom, i.e.,

$$
\begin{gathered}
\operatorname{supp} \tilde{t}_{i, k} \subseteq \operatorname{supp} t_{i, k}, \quad i=1, \ldots, 5, \quad k \in \mathbb{Z}^{2}, \\
\tilde{t}_{i, k}=\tilde{t}_{i}(\cdot-k) \quad i=1,2,3, \quad k \in \mathbb{Z}^{2}, \\
\tilde{t}_{4, k}= \begin{cases}\tilde{t}_{4}^{+}(\cdot-k), & \text { if } k \in \mathbb{Z} \times 2 \mathbb{Z}, \\
\tilde{t}_{4}^{-}(\cdot-k+(0,-1)), & \text { if } k \in \mathbb{Z} \times(2 \mathbb{Z}-1),\end{cases} \\
\tilde{t}_{5, k}= \begin{cases}\tilde{t}_{5}^{+}(\cdot-k), & \text { if } k \in 2 \mathbb{Z} \times \mathbb{Z}, \\
\tilde{t}_{5}^{-}(\cdot-k+(-1,0)), & \text { if } k \in(2 \mathbb{Z}-1) \times \mathbb{Z},\end{cases} \\
\tilde{t}_{3}\left(x_{1}, x_{2}\right)=\tilde{t}_{2}\left(-x_{1}, x_{2}\right), \\
\tilde{t}_{5}^{+}\left(x_{1}, x_{2}\right)=\tilde{t}_{4}^{+}\left(x_{2}, x_{1}\right), \quad \tilde{t}_{5}^{-}\left(x_{1}, x_{2}\right)=\tilde{t}_{4}^{-}\left(x_{2}, x_{1}\right), \\
\tilde{t}_{1}\left(x_{1}, x_{2}\right)=\tilde{t}_{1}\left(x_{2}, x_{1}\right)=\tilde{t}_{1}\left(\left|x_{1}\right|,\left|x_{2}\right|\right), \\
\tilde{t}_{2}\left(x_{1}, x_{2}\right)=\tilde{t}_{2}\left(-x_{2},-x_{1}\right)=-\tilde{t}_{2}\left(x_{2}, x_{1}\right), \\
\tilde{t}_{4}^{+}\left(x_{1}, x_{2}\right)=\tilde{t}_{4}^{+}\left(x_{1},-x_{2}\right)=-\tilde{t}_{4}^{+}\left(-x_{1}, x_{2}\right), \\
\tilde{t}_{4}^{-}\left(x_{1}, x_{2}\right)=-\tilde{t}_{4}^{-}\left(-x_{1}, x_{2}\right)=-\tilde{t}_{4}^{-}\left(x_{1},-x_{2}\right),
\end{gathered}
$$

where we set

$$
\begin{aligned}
\tilde{t}_{i}:=\tilde{t}_{i,(0,0)}, & i=1,2,3, \\
\tilde{t}_{4}^{+}:=\tilde{t}_{4,(0,0)}, & \tilde{t}_{4}^{-}:=\tilde{t}_{4,(0,-1)}, \\
\tilde{t}_{5}^{+}:=\tilde{t}_{5,(0,0)}, & \tilde{t}_{5}^{-}:=\tilde{t}_{5,(-1,0)} .
\end{aligned}
$$

In addition, to remove the still remaining degrees of freedom, we require that

$$
\left.\begin{array}{l}
\operatorname{supp} \tilde{t}_{1} \\
\operatorname{supp} \tilde{t}_{4}^{+} \\
\operatorname{supp} \tilde{t}_{4}^{-}
\end{array}\right\} \subseteq\left\{\left(x_{1}, x_{2}\right) \in \mathbb{R}^{2}:\left|x_{1}\right| \leq 1,\left|x_{2}\right| \leq 1,\left|x_{1} \pm x_{2}\right| \leq 3 / 2\right\}
$$

and

$$
D_{x_{1}} \tilde{t}_{4}^{+}(0,0)=D_{x_{2}} \tilde{t}_{4}^{-}\left(\frac{1}{2}, 0\right)=D_{x_{2}} \tilde{t}_{4}^{-}\left(\frac{3}{4}, 0\right)=0 .
$$

Note that (3.7) is natural to assume since $t_{4}^{+}$and $t_{4}^{-}$have the corresponding properties.

Theorem 3.1. There is a unique set of biorthogonal dual functions $\tilde{\mathcal{B}}=\left\{\tilde{t}_{i, k}: i=\right.$ $\left.1, \ldots, 5, k \in \mathbb{Z}^{2}\right\} \subset S_{3}^{1}\left(\frac{1}{2} \triangle\right)$ satisfying (3.1)-(3.7).

Proof: It is sufficient to establish the existence and uniqueness of the functions $\tilde{t}_{1}, \tilde{t}_{2}, \tilde{t}_{4}^{+}$ and $\tilde{t}_{4}^{-}$satisfying (3.1), (3.2), (3.5)-(3.7) since the other basis functions can then be defined using (3.3) and (3.4) and necessarily satisfy all desired conditions. 
The basis function $\tilde{t}_{1}$, if exists, must satisfy $\operatorname{supp} \tilde{t}_{1} \subseteq[-1,1]^{2}$. Therefore, by the support and local linear independence properties of $\mathcal{B}_{\mathrm{FE}}\left(\frac{1}{2} \triangle\right), \tilde{t}_{1}$ is a linear combination of functions $s_{i, k}(2 \cdot)$ with $\operatorname{supp} s_{i, k}(2 \cdot) \subseteq[-1,1]^{2}$. Moreover, by symmetry (3.5) we conclude that $\tilde{t}_{1}$ has the following form:

$$
\begin{aligned}
\tilde{t}_{1}(\cdot / 2)= & a_{1} s_{1} \\
& +a_{2}\left(s_{1,(1,0)}+s_{1,(0,1)}+s_{1,(-1,0)}+s_{1,(0,-1)}\right) \\
& +a_{3}\left(s_{1,(1,1)}+s_{1,(-1,1)}+s_{1,(1,-1)}+s_{1,(-1,-1)}\right) \\
+ & a_{4}\left(s_{2,(-1,0)}-s_{2,(1,0)}+s_{3,(0,-1)}-s_{3,(0,1)}\right) \\
+ & a_{5}\left(s_{2,(1,1)}+s_{3,(1,1)}+s_{2,(1,-1)}-s_{3,(1,-1)}\right. \\
& \left.\quad-s_{2,(-1,1)}+s_{3,(-1,1)}-s_{2,(-1,-1)}-s_{3,(-1,-1)}\right) \\
+ & a_{6}\left(s_{4,(1,0)}-s_{4,(-1,0)}+s_{5,(0,1)}-s_{5,(0,-1)}\right. \\
& \left.\quad+s_{4,(1,-1)}-s_{4,(-1,-1)}+s_{5,(-1,1)}-s_{5,(-1,-1)}\right) \\
+ & a_{7}\left(s_{4,(1,1)}-s_{4,(-1,1)}+s_{5,(1,1)}-s_{5,(1,-1)}\right. \\
& \left.\quad+s_{4,(1,-2)}-s_{4,(-1,-2)}+s_{5,(-2,1)}-s_{5,(-2,-1)}\right)
\end{aligned}
$$

with some real coefficients $a_{1}, \ldots, a_{7}$. Due to the symmetry of $\tilde{t}_{1},(3.1)$ is equivalent to the following six conditions:

$$
\begin{aligned}
& \left\langle\tilde{t}_{1}, t_{1}\right\rangle=\left\langle t_{1}, t_{1}\right\rangle, \quad\left\langle\tilde{t}_{1}, t_{1,(1,0)}\right\rangle=\left\langle\tilde{t}_{1}, t_{1,(1,1)}\right\rangle=0 \\
& \left\langle\tilde{t}_{1}, t_{2,(1,0)}\right\rangle=\left\langle\tilde{t}_{1}, t_{2,(1,-1)}\right\rangle=\left\langle\tilde{t}_{1}, t_{4,(1,0)}\right\rangle=0 .
\end{aligned}
$$

We note that the symmetry ensures that all other orthogonality conditions are satisfied, e.g. $\left\langle\tilde{t}_{1}, t_{4}\right\rangle=0$ since the integrand is odd in $x_{1}$.

By considering the Bernstein-Bézier coefficients of the finite element basis functions $s_{i, k}$ it is not difficult to see that $\tilde{t}_{1}$ satisfies (3.6) if and only if

$$
a_{3} / 4+a_{5} / 24+a_{7} / 6=0
$$

This gives us a total of seven equations to determine the seven coefficients $a_{1}, \ldots, a_{7}$. Using Matlab we find that this system of linear equations has a unique solution

$$
\begin{array}{lll}
a_{1}=\frac{12698499}{2576168}, & a_{2}=-\frac{4556039}{5152336}, & a_{3}=\frac{2117951}{10304672}, \\
a_{5}=-\frac{1732869}{2576168}, & a_{6}=-\frac{35636205}{20609344}, & a_{7}=-\frac{2888115}{20609344} .
\end{array}
$$


Similarly, we have by symmetry assumptions and the support property of $\mathcal{B}_{\mathrm{FE}}\left(\frac{1}{2} \triangle\right)$,

$$
\begin{aligned}
\tilde{t}_{2}(\cdot / 2)= & b_{1}\left(s_{1,(1,0)}-s_{1,(-1,0)}+s_{1,(0,-1)}-s_{1,(0,1)}\right) \\
& +b_{2}\left(s_{2}-s_{3}\right) \\
& +b_{3}\left(s_{2,(0,1)}+s_{2,(0,-1)}-s_{3,(1,0)}-s_{3,(-1,0)}\right) \\
& +b_{4}\left(s_{2,(1,0)}+s_{2,(-1,0)}-s_{3,(0,1)}-s_{3,(0,-1)}\right) \\
& +b_{5}\left(s_{2,(1,1)}+s_{2,(-1,-1)}-s_{3,(1,1)}-s_{3,(-1,-1)}\right) \\
& +b_{6}\left(s_{4}+s_{4,(0,-1)}-s_{5}-s_{5,(-1,0)}\right) \\
& +b_{7}\left(s_{4,(1,0)}+s_{4,(-1,-1)}-s_{5,(0,1)}-s_{5,(-1,-1)}\right) \\
& +b_{8}\left(s_{4,(0,1)}+s_{4,(0,-2)}-s_{5,(1,0)}-s_{5,(-2,0)}\right) \\
& +b_{9}\left(s_{4,(1,1)}+s_{4,(-1,-2)}-s_{5,(1,1)}-s_{5,(-2,-1)}\right) \\
& +b_{10}\left(s_{4,(-1,0)}+s_{4,(1,-1)}-s_{5,(0,-1)}-s_{5,(-1,1)}\right) .
\end{aligned}
$$

By considering the Bernstein-Bézier coefficients of the basis functions $s_{i, k}$, we see that $\tilde{t}_{2}$ satisfies (3.2) if and only if

$$
\begin{gathered}
b_{1} / 2-b_{3} / 12+b_{4} / 6+b_{8} / 3=0, \\
b_{1}+b_{3} / 12+b_{4} / 12+b_{8} / 3+b_{10} / 3=0 .
\end{gathered}
$$

Nontrivial biorthogonality conditions are:

$$
\begin{aligned}
& \left\langle\tilde{t}_{2}, t_{1,(1,0)}\right\rangle=0, \quad\left\langle\tilde{t}_{2}, t_{2}\right\rangle=\left\langle t_{2}, t_{2}\right\rangle \\
& \left\langle\tilde{t}_{2}, t_{2,(1,0)}\right\rangle=\left\langle\tilde{t}_{2}, t_{2,(1,1)}\right\rangle=\left\langle\tilde{t}_{2}, t_{3,(1,0)}\right\rangle=0 \\
& \left\langle\tilde{t}_{2}, t_{4,(0,0)}\right\rangle=\left\langle\tilde{t}_{2}, t_{4,(1,0)}\right\rangle=\left\langle\tilde{t}_{2}, t_{4,(1,-1)}\right\rangle=0 .
\end{aligned}
$$

Again, a computation with Matlab finds that the resulting system of 10 equations with 10 unknowns is nonsingular, and the $b_{i}$ 's are given by

$$
\begin{array}{llll}
b_{1}=-\frac{112135}{505064}, & b_{2}=\frac{9539839}{1136394}, & b_{3}=\frac{484907}{505064}, & b_{4}=\frac{2567543}{2272788}, \\
b_{5}=\frac{4141}{17416}, & b_{6}=-\frac{40839731}{18182304}, & b_{7}=-\frac{47560123}{418192992}, & b_{8}=\frac{149281}{18182304}, \\
b_{9}=\frac{259927495}{418192992}, & b_{10}=\frac{1231025}{9091152} . & &
\end{array}
$$


Similar considerations show that $\tilde{t}_{4}^{+}$has the form

$$
\begin{aligned}
\tilde{t}_{4}^{+}(\cdot / 2)= & c_{1}\left(s_{1,(1,0)}-s_{1,(-1,0)}\right) \\
& +c_{2}\left(s_{1,(1,1)}-s_{1,(-1,1)}+s_{1,(1,-1)}-s_{1,(-1,-1)}\right) \\
& +c_{3} s_{2} \\
& +c_{4}\left(s_{2,(1,0)}+s_{2,(-1,0)}\right) \\
& +c_{5}\left(s_{2,(0,1)}+s_{2,(0,-1)}\right) \\
& +c_{6}\left(s_{2,(1,1)}+s_{2,(-1,1)}+s_{2,(1,-1)}+s_{2,(-1,-1)}\right) \\
& +c_{7}\left(s_{3,(1,1)}-s_{3,(-1,1)}-s_{3,(1,-1)}+s_{3,(-1,-1)}\right) \\
& +c_{8}\left(s_{4}+s_{4,(0,-1)}\right) \\
& +c_{9}\left(s_{4,(0,1)}+s_{4,(0,-2)}\right) \\
& +c_{10}\left(s_{4,(1,0)}+s_{4,(1,-1)}+s_{4,(-1,0)}+s_{4,(-1,-1)}\right) \\
& +c_{11}\left(s_{4,(1,1)}+s_{4,(-1,1)}+s_{4,(1,-2)}+s_{4,(-1,-2)}\right) \\
& +c_{12}\left(s_{5,(0,1)}-s_{5,(0,-1)}-s_{5,(-1,1)}+s_{5,(-1,-1)}\right) \\
& +c_{13}\left(s_{5,(1,1)}-s_{5,(1,-1)}-s_{5,(-2,1)}+s_{5,(-2,-1)}\right) .
\end{aligned}
$$

It is easy to check that $\tilde{t}_{4}^{+}$satisfies (3.6) if and only if

$$
\begin{gathered}
c_{2} / 2-c_{6} / 12+c_{7} / 6+c_{11} / 3=0, \\
c_{2}+c_{6} / 12+c_{7} / 12+c_{11} / 3+c_{13} / 3=0
\end{gathered}
$$

and it satisfies (3.7) if and only if

$$
c_{3}=0
$$

In addition, we have 10 nontrivial biorthogonality conditions:

$$
\begin{gathered}
\left\langle\tilde{t}_{4}^{+}, t_{1,(1,0)}\right\rangle=\left\langle\tilde{t}_{4}^{+}, t_{1,(1,1)}\right\rangle=0, \\
\left\langle\tilde{t}_{4}^{+}, t_{2}\right\rangle=\left\langle\tilde{t}_{4}^{+}, t_{2,(1,0)}\right\rangle=\left\langle\tilde{t}_{4}^{+}, t_{2,(0,1)}\right\rangle=\left\langle\tilde{t}_{4}^{+}, t_{2,(1,1)}\right\rangle=\left\langle\tilde{t}_{4}^{+}, t_{2,(1,-1)}\right\rangle=0, \\
\left\langle\tilde{t}_{4}^{+}, t_{4}^{+}\right\rangle=\left\langle t_{4}^{+}, t_{4}^{+}\right\rangle, \quad\left\langle\tilde{t}_{4}^{+}, t_{4,(1,0)}\right\rangle=\left\langle\tilde{t}_{4}^{+}, t_{5,(-1,1)}\right\rangle=0 .
\end{gathered}
$$

This gives

$$
\begin{array}{llll}
c_{1}=\frac{53506383}{196402666}, & c_{2}=-\frac{931930406}{2062227993}, & c_{3}=0, & c_{4}=-\frac{20270664283}{2749637324}, \\
c_{5}=\frac{12775765033}{1374818662}, & c_{6}=\frac{6848898731}{5499274648}, & c_{7}=\frac{9417522901}{5499274648}, & c_{8}=\frac{8042814861}{5499274648}, \\
c_{9}=\frac{2388717689}{5499274648}, & c_{10}=\frac{222303404979}{21997098592}, & c_{11}=\frac{2924739425}{21997098592}, & c_{12}=-\frac{2846476149}{21997098592}, \\
c_{13}=\frac{10630611935}{21997098592} . & & &
\end{array}
$$


Next, $\tilde{t}_{4}^{-}$has the form

$$
\begin{aligned}
\tilde{t}_{4}^{-}(\cdot / 2)= & d_{1}\left(s_{1,(1,1)}-s_{1,(-1,1)}-s_{1,(1,-1)}+s_{1,(-1,-1)}\right) \\
& +d_{2}\left(s_{2,(0,1)}-s_{2,(0,-1)}\right) \\
& +d_{3}\left(s_{2,(1,1)}+s_{2,(-1,1)}-s_{2,(1,-1)}-s_{2,(-1,-1)}\right) \\
& +d_{4}\left(s_{3,(1,0)}-s_{3,(-1,0)}\right) \\
& +d_{5}\left(s_{3,(1,1)}-s_{3,(-1,1)}+s_{3,(1,-1)}-s_{3,(-1,-1)}\right) \\
& +d_{6}\left(s_{4}-s_{4,(0,-1)}\right) \\
& +d_{7}\left(s_{4,(0,1)}-s_{4,(0,-2)}\right) \\
& +d_{8}\left(s_{4,(1,0)}+s_{4,(-1,0)}-s_{4,(1,-1)}-s_{4,(-1,-1)}\right) \\
& +d_{9}\left(s_{4,(1,1)}+s_{4,(-1,1)}-s_{4,(1,-2)}-s_{4,(-1,-2)}\right) \\
& +d_{10}\left(s_{5}-s_{5,(-1,0)}\right) \\
& +d_{11}\left(s_{5,(1,0)}-s_{5,(-2,0)}\right) \\
& +d_{12}\left(s_{5,(0,1)}+s_{5,(0,-1)}-s_{5,(-1,1)}-s_{5,(-1,-1)}\right) \\
& +d_{13}\left(s_{5,(1,1)}+s_{5,(1,-1)}-s_{5,(-2,1)}-s_{5,(-2,-1)}\right),
\end{aligned}
$$

where

$$
\begin{gathered}
d_{1} / 2-d_{3} / 12+d_{5} / 6+d_{9} / 3=0 \\
d_{1}+d_{3} / 12+d_{5} / 12+d_{9} / 3+d_{13} / 3=0 \\
d_{4}=d_{11}=0
\end{gathered}
$$

to ensure (3.6) and (3.7), and

$$
\begin{gathered}
\left\langle\tilde{t}_{4}^{-}, t_{1,(1,1)}\right\rangle=0, \\
\left\langle\tilde{t}_{4}^{-}, t_{2,(1,0)}\right\rangle=\left\langle\tilde{t}_{4}^{-}, t_{2,(0,1)}\right\rangle=\left\langle\tilde{t}_{4}^{-}, t_{2,(1,1)}\right\rangle=\left\langle\tilde{t}_{4}^{-}, t_{2,(1,-1)}\right\rangle=0, \\
\left\langle\tilde{t}_{4}^{-}, t_{4}^{-}\right\rangle=\left\langle t_{4}^{-}, t_{4}^{-}\right\rangle, \quad\left\langle\tilde{t}_{4}^{-}, t_{4,(1,-1)}\right\rangle=\left\langle\tilde{t}_{4}^{-}, t_{5,(-1,0)}\right\rangle=\left\langle\tilde{t}_{4}^{-}, t_{5,(-1,1)}\right\rangle=0,
\end{gathered}
$$

to guarantee the biorthogonality.

The linear system is again uniquely solvable, with

$$
\begin{array}{llll}
d_{1}=-\frac{160587713}{3629286668}, & d_{2}=\frac{2810669524}{907321667}, & d_{3}=\frac{458590743}{1814643334}, & d_{4}=0, \\
d_{5}=\frac{66969045}{1814643334}, & d_{6}=-\frac{5548921443}{907321667}, & d_{7}=\frac{1993454073}{259234762}, & d_{8}=\frac{588629171}{3629286668}, \\
d_{9}=\frac{14400282}{129617381}, & d_{10}=-\frac{998583157}{259234762}, & d_{11}=0, & d_{12}=-\frac{13573487031}{1814643334}, \\
d_{13}=-\frac{184224651}{3629286668} . & & &
\end{array}
$$

Clearly, $\tilde{t}_{3}, \tilde{t}_{5}^{+}$and $\tilde{t}_{5}^{-}$are to be constructed using the same ideas from (3.4),

$$
\tilde{t}_{3}\left(x_{1}, x_{2}\right)=\tilde{t}_{2}\left(-x_{1}, x_{2}\right), \quad \tilde{t}_{5}^{ \pm}\left(x_{1}, x_{2}\right)=\tilde{t}_{4}^{ \pm}\left(x_{2}, x_{1}\right),
$$

and satisfy all our requirements. 
Remark 3.2. It is impossible to construct a function $\tilde{s}_{4} \in S_{3}^{1}\left(\frac{1}{2} \triangle\right)$ with the property supp $\tilde{s}_{4} \subseteq \operatorname{supp} s_{4}$ such that $\tilde{s}_{4}$ satisfy the biorthogonality conditions with respect to a basis for $S_{3}^{1}(\triangle)$ that includes $s_{4}$. Indeed, there are only 19 linear independent functions in $S_{3}^{1}\left(\frac{1}{2} \triangle\right)$ whose supports are subsets of $\operatorname{supp} s_{4}=[-1,1] \times[0,1]$, whereas $\left.\operatorname{dim} S_{3}^{1}(\triangle)\right|_{[-1,1] \times[0,1]}=25$. Similar arguments apply to $s_{5}$. This fact was our primary reason to replace $s_{4}, s_{5}$ with $t_{4}^{ \pm}, t_{5}^{ \pm}$via a Haar-like transform (2.5).

Theorem 3.3. The set

$$
\tilde{\mathcal{B}} \cup\left\{t_{i, k}(2 \cdot): k \in \mathbb{Z}^{2} \backslash \mathcal{Z}_{i}, \quad i=1, \ldots, 5\right\},
$$

where

$$
\begin{array}{cl}
\mathcal{Z}_{i}:=2 \mathbb{Z}^{2}, & i=1,2,3, \\
\mathcal{Z}_{4}:=2 \mathbb{Z} \times(4 \mathbb{Z} \cup(4 \mathbb{Z}-1)), \quad \mathcal{Z}_{5}:=(4 \mathbb{Z} \cup(4 \mathbb{Z}-1)) \times 2 \mathbb{Z},
\end{array}
$$

is a Riesz basis for the space $S_{3}^{1}\left(\frac{1}{2} \triangle\right)$.

Proof: In view of the transformation formulas between $\mathcal{B}$ and $\mathcal{B}_{\mathrm{FE}}$ (see Section 2.2), it suffices to show that

$$
\tilde{\mathcal{B}} \cup\left\{s_{i, k}(2 \cdot),: k \in \mathbb{Z}^{2} \backslash \mathcal{Z}_{i}, \quad i=1, \ldots, 5\right\}
$$

is a Riesz basis for $S_{3}^{1}\left(\frac{1}{2} \triangle\right)$.

Let

$$
s=\sum_{i=1}^{5} \sum_{k \in \mathbb{Z}^{2}} \tilde{\alpha}_{i, k} \tilde{t}_{i, k}+\sum_{i=1}^{5} \sum_{k \in \mathbb{Z}^{2} \backslash \mathcal{Z}_{i}} \alpha_{i, k} s_{i, k}(2 \cdot),
$$

such that

$$
\|\alpha\|_{2}^{2}=\sum_{i=1}^{5} \sum_{k \in \mathbb{Z}^{2}}\left|\tilde{\alpha}_{i, k}\right|^{2}+\sum_{i=1}^{5} \sum_{k \in \mathbb{Z}^{2} \backslash \mathcal{Z}_{i}}\left|\alpha_{i, k}\right|^{2}<\infty
$$

We have

$$
\|s\|_{2}^{2}=\int_{\mathbf{R}^{2}}|s|^{2}=\sum_{m \in 2 \mathbb{Z}^{2}} \int_{[-1,1]^{2}+m}|s|^{2} .
$$

Given any $m \in 2 \mathbb{Z}^{2}$, we consider the space $\left.S_{3}^{1}\left(\frac{1}{2} \triangle\right)\right|_{[-1,1]^{2}+m}$ of splines in $S_{3}^{1}\left(\frac{1}{2} \triangle\right)$ restricted to $[-1,1]^{2}+m$. It is not difficult to see that

$$
\left.\operatorname{dim} S_{3}^{1}\left(\frac{1}{2} \triangle\right)\right|_{[-1,1]^{2}+m}=115,
$$

and the following splines form a basis for $\left.S_{3}^{1}\left(\frac{1}{2} \triangle\right)\right|_{[-1,1]^{2}+m}$ :

$$
\begin{array}{ll}
s_{i, k+2 m}(2 \cdot)=s_{i, k}(2 \cdot-2 m), & i=1,2,3, \quad k \in \mathbb{Z}^{2} \cap[-2,2]^{2}, \\
s_{4, k+2 m}(2 \cdot)=s_{4, k}(2 \cdot-2 m), & k \in \mathbb{Z}^{2} \cap([-2,2] \times[-2,1]), \\
s_{5, k+2 m}(2 \cdot)=s_{5, k}(2 \cdot-2 m), & k \in \mathbb{Z}^{2} \cap([-2,1] \times[-2,2]) .
\end{array}
$$


We now replace some of these basis functions with splines in $\tilde{\mathcal{B}}$ and consider the following set of splines of the same cardinality 115,

$$
\begin{array}{ll}
\tilde{t}_{i, k}(\cdot-m), & i=1,2,3, \quad k \in \mathbb{Z}^{2} \cap[-1,1]^{2}, \\
\tilde{t}_{4, k}(\cdot-m), & k \in \mathbb{Z}^{2} \cap([-1,1] \times[-1,0]), \\
\tilde{t}_{5, k}(\cdot-m), & k \in \mathbb{Z}^{2} \cap([-1,0] \times[-1,1]), \\
s_{i, k}(2 \cdot-2 m), & i=1,2,3, \quad k \in\left(\mathbb{Z}^{2} \backslash 2 \mathbb{Z}^{2}\right) \cap[-2,2]^{2}, \\
s_{4, k}(2 \cdot-2 m), & k \in\left(\mathbb{Z}^{2} \backslash \mathcal{Z}_{4}\right) \cap([-2,2] \times[-2,1]), \\
s_{5, k}(2 \cdot-2 m), & k \in\left(\mathbb{Z}^{2} \backslash \mathcal{Z}_{5}\right) \cap([-2,1] \times[-2,2]) .
\end{array}
$$

We claim that this set is also a basis for $\left.S_{3}^{1}\left(\frac{1}{2} \triangle\right)\right|_{[-1,1]^{2}+m}$. Indeed, the matrix of the transformation of the basis (3.10) into the system (3.11) is given by

$$
M=\left[\begin{array}{cc}
A & B \\
O & I_{76}
\end{array}\right]
$$

where $I_{n}$ denotes the $n \times n$ identity matrix, $O$ a zero matrix, $B$ a $39 \times 76$ matrix, and

$$
A=\left[\begin{array}{ccccc}
a_{1} I_{9} & \star & \star & \star & \star \\
O & b_{2} I_{9} & -b_{2} I_{9} & \star & \star \\
O & -b_{2} I_{9} & -b_{2} I_{9} & \star & \star \\
O & O & O & C & D \\
O & O & O & D & C
\end{array}\right]
$$

with

$$
C=\left[\begin{array}{cc}
c_{8} I_{3} & c_{8} I_{3} \\
d_{6} I_{3} & -d_{6} I_{3}
\end{array}\right], \quad D=\left[\begin{array}{cccccc}
0 & 0 & 0 & 0 & 0 & 0 \\
0 & 0 & 0 & 0 & 0 & 0 \\
0 & 0 & 0 & 0 & 0 & 0 \\
d_{10} & 0 & 0 & -d_{10} & 0 & 0 \\
0 & 0 & 0 & 0 & 0 & 0 \\
0 & 0 & 0 & 0 & 0 & 0
\end{array}\right]
$$

Here the coefficients $a_{1}, b_{2}$ etc. are as in the definitions of $\tilde{t}_{1}, \tilde{t}_{2}$ etc. A simple computation shows that

$$
\operatorname{det} A=a_{1}^{9}\left(-2 b_{2}\right)^{9} \operatorname{det}\left[\begin{array}{cc}
C & D \\
D & C
\end{array}\right]=-2^{15} a_{1}^{9} b_{2}^{9} c_{8}^{6} d_{6}^{4}\left(d_{6}^{2}-d_{10}^{2}\right)
$$

Since $a_{1}, b_{2}, c_{8}, d_{6}$ are nonzero, and

$$
d_{6}^{2}-d_{10}^{2}=\frac{74300868971580563195}{3292930429630635556} \neq 0
$$

the matrix $M$ is nonsingular, which proves our claim. 
Since the system (3.11) is precisely the set of all splines in (3.9) whose supports have nonempty intersection with the interior of $[-1,1]^{2}+m$, and since each function in (3.9) is supported on at most four of the sets $[-1,1]^{2}+m, m \in 2 \mathbb{Z}^{2}$, we get the inequality

$$
C_{1}^{2}\|\alpha\|_{2}^{2} \leq \sum_{m \in 2 \mathbb{Z}^{2}} \int_{[-1,1]^{2}+m}|s|^{2} \leq 4 C_{2}^{2}\|\alpha\|_{2}^{2},
$$

where $C_{1}, C_{2}>0$ are the Riesz constants of the (finite) basis (3.11). (Obviously, $C_{1}$ and $C_{2}$ are independent of $m$.) Thus,

$$
C_{1}\|\alpha\|_{2} \leq\|s\|_{2} \leq 2 C_{2}\|\alpha\|_{2}
$$

and the proof is complete.

\section{$\S 4$. Prewavelets}

Let for all $i=1, \ldots, 5, k \in \mathbb{Z}^{2} \backslash \mathcal{Z}_{i}$,

$$
\psi_{i, k}:=t_{i, k}(2 \cdot)-\sum_{j=1}^{5} \sum_{\ell \in \mathbb{Z}^{2}} \frac{\left\langle t_{i, k}(2 \cdot), t_{j, \ell}\right\rangle}{\left\|t_{j, \ell}\right\|_{2}^{2}} \tilde{t}_{j, \ell}
$$

Note that, due to the local support of the functions $t_{i, k}$ and $\tilde{t}_{j, \ell}$, the above sums have only finite number (at most 39, in fact) of nonzero terms. Consequently, the support of each function $\psi_{i, k}$ is contained in a square of sidelength four.

By using the biorthogonality conditions it is easy to see that the functions $\psi_{i, k}$ are orthogonal to the basis splines of $\mathcal{B}$, and therefore they lie in the prewavelet space

$$
W_{1}:=S_{3}^{1}\left(\frac{1}{2} \triangle\right) \ominus S_{3}^{1}(\triangle)
$$

Moreover,

$$
W_{1}=\operatorname{span}_{\ell^{2}\left(\mathbb{Z}^{2}\right)}\left\{\psi_{i, k}: i=1, \ldots, 5, k \in \mathbb{Z}^{2} \backslash \mathcal{Z}_{i}\right\} .
$$

Indeed, let $s \in W_{1}$. Since $W_{1} \subset S_{3}^{1}\left(\frac{1}{2} \triangle\right)$, by Theorem 3.3 there exists a unique representation

$$
s=\sum_{i=1}^{5} \sum_{k \in \mathbb{Z}^{2} \backslash \mathcal{Z}_{i}} \alpha_{i, k} t_{i, k}(2 \cdot)+\sum_{j=1}^{5} \sum_{\ell \in \mathbb{Z}^{2}} \tilde{\alpha}_{j, \ell} \tilde{t}_{j, \ell}
$$

with square summable coefficients. Since the basis functions have local support, we compute the scalar products $\left\langle s, t_{j, \ell}\right\rangle=0$ termwise and get

$$
\tilde{\alpha}_{j, \ell}=-\sum_{i=1}^{5} \sum_{k \in \mathbb{Z}^{2} \backslash \mathcal{Z}_{i}} \alpha_{i, k} \frac{\left\langle t_{i, k}(2 \cdot), t_{j, \ell}\right\rangle}{\left\|t_{j, \ell}\right\|_{2}^{2}}, \quad j=1, \ldots, 5, \quad \ell \in \mathbb{Z}^{2}
$$


which implies

$$
s=\sum_{i=1}^{5} \sum_{k \in \mathbb{Z}^{2} \backslash \mathcal{Z}_{i}} \alpha_{i, k} \psi_{i, k}
$$

Lemma 4.1. The functions (4.1) form a Riesz basis for $W_{1}$.

Proof: Given $\alpha_{i, k} \in \mathbb{R}, i=1, \ldots, 5, k \in \mathbb{Z}^{2} \backslash \mathcal{Z}_{i}$, let

$$
\begin{aligned}
s & =\sum_{i=1}^{5} \sum_{k \in \mathbb{Z}^{2} \backslash \mathcal{Z}_{i}} \alpha_{i, k} \psi_{i, k} \\
& =\sum_{i=1}^{5} \sum_{k \in \mathbb{Z}^{2} \backslash \mathcal{Z}_{i}} \alpha_{i, k} t_{i, k}(2 \cdot)-\sum_{j=1}^{5} \sum_{\ell \in \mathbb{Z}^{2}}\left(\sum_{i=1}^{5} \sum_{k \in \mathbb{Z}^{2} \backslash \mathcal{Z}_{i}} \alpha_{i, k} \frac{\left\langle t_{i, k}(2 \cdot), t_{j, \ell}\right\rangle}{\left\|t_{j, \ell}\right\|_{2}^{2}}\right) \tilde{t}_{j, \ell} .
\end{aligned}
$$

By Theorem 3.3 we immediately get the lower estimate

$$
\|s\|_{2} \geq C_{1}\|\alpha\|_{2}
$$

where $C_{1}>0$ is the absolute constant from (3.12). On the other hand, since at most 115 scalar products $\left\langle t_{i, k}(2 \cdot), t_{j, \ell}\right\rangle$ are nonzero for fixed $j, \ell$, and at most 39 of them are nonzero for fixed $i, k$, we have by a standard argument

$$
\sum_{j=1}^{3} \sum_{\ell \in \mathbb{Z}^{2}}\left|\sum_{i=1}^{5} \sum_{k \in \mathbb{Z}^{2} \backslash \mathcal{Z}_{i}} \alpha_{i, k} \frac{\left\langle t_{i, k}(2 \cdot), t_{j, \ell}\right\rangle}{\left\|t_{j, \ell}\right\|_{2}^{2}}\right|^{2} \leq C_{3}\|\alpha\|_{2}^{2}
$$

where

$$
C_{3}=115 \cdot 39 \cdot \frac{1}{4} \max _{\substack{i, j=1, \ldots, 5 \\ k, \ell \in \mathbb{Z}^{2}}} \frac{\left\|t_{i, k}\right\|_{2}^{2}}{\left\|t_{j, \ell}\right\|_{2}^{2}}<\infty
$$

is a finite constant since $t_{i, k}$ are translates of only 7 functions $t_{1}, t_{2}, t_{3}, t_{4}^{ \pm}, t_{5}^{ \pm}$. Therefore, by Theorem 3.3

$$
\|s\|_{2} \leq C_{4}\|\alpha\|_{2},
$$

where $C_{4}>0$ is an absolute constant. The proof is complete.

Theorem 4.2. The functions

$$
\psi_{i, k}^{[j]}:=2^{j-1} \psi_{i, k}\left(2^{j-1} \cdot\right), \quad i=1,2,3,4,5, \quad k \in \mathbb{Z}^{2} \backslash \mathcal{Z}_{i}, \quad j \in \mathbb{Z}
$$

form a prewavelet basis for $L^{2}\left(\mathbb{R}^{2}\right)$.

Proof: Indeed, by Lemma 4.1 for all $j \in \mathbb{Z}$,

$$
\psi_{i, k}^{[j]}:=2^{j} \psi_{i, k}\left(2^{j} \cdot\right), \quad i=1,2,3,4,5, \quad k \in \mathbb{Z}^{2} \backslash \mathcal{Z}_{i},
$$


is a Riesz basis for $W_{j}:=S_{3}^{1}\left(2^{-j} \triangle\right) \ominus S_{3}^{1}\left(2^{-j+1} \triangle\right)$ with the same Riesz constants $C_{1}, C_{4}$. Since we have orthogonal decomposition

$$
L^{2}\left(\mathbb{R}^{2}\right)=\bigoplus_{j \in \mathbb{Z}} W_{j}
$$

the statement follows.

Finally, we note that our prewavelets $\psi_{i, k}$ can be obtained as translates of a finite subset of them. Indeed, by (2.6) it is easy to see that

$$
\left\{t_{i, k}(2 \cdot): k \in \mathbb{Z}^{2} \backslash \mathcal{Z}_{i}\right\}=\left\{t_{i, k}(2 \cdot-2 m): k=(1,0),(0,1),(1,1), \quad m \in \mathbb{Z}^{2}\right\},
$$

for $i=1,2,3$, and

$$
\begin{aligned}
& \left\{t_{4, k}(2 \cdot): k \in \mathbb{Z}^{2} \backslash \mathcal{Z}_{4}\right\}=\left\{t_{4, k}^{ \pm}(2 \cdot-2 m): k=(1,0),(0,2),(1,2), \quad m \in \mathbb{Z} \times 2 \mathbb{Z}\right\}, \\
& \left\{t_{5, k}(2 \cdot): k \in \mathbb{Z}^{2} \backslash \mathcal{Z}_{5}\right\}=\left\{t_{5, k}^{ \pm}(2 \cdot-2 m): k=(0,1),(2,0),(2,1), \quad m \in 2 \mathbb{Z} \times \mathbb{Z}\right\},
\end{aligned}
$$

for $i=4,5$. In view of (4.1), this implies

$$
\psi_{i, k+2 m}=\psi_{i, k}(\cdot-m), \quad m \in 2 \mathbb{Z}^{2} .
$$

We note, however, that (4.1) is the closed form (where everything is known once the inner products are computed) to be used in practical applications due to its simplicity (and the inner products are computed only once and for all).

Remark 4.3. Stability in Sobolev spaces $\mathcal{H}^{s}$ : It can be shown by a standard argument (see e.g. [12]) that the prewavelets (4.2) form a stable basis of the Sobolev spaces $\mathcal{H}^{s}$ for all $-5 / 2<s<5 / 2$.

Remark 4.4. Prewavelets on bounded domains in $\mathbb{R}^{2}$ : The above construction can be employed to construct prewavelets on bounded domains due to their own local support and the explicit construction we use. This is also not possible when box spline constructions are used where always the whole domain $\mathbb{R}^{2}$ has to be incorporated.

Remark 4.5. Irregular quadrangulations: Unlike the usual constructions, e.g. in [1], this construction is without the use of Fourier transforms and can therefore, in principle, be extended to irregular quadrangulations by pertubation arguments and re-computation of the explicit coefficients. The analogs of bases $\mathcal{B}_{\mathrm{FE}}$ and $\mathcal{B}$ are readily available for any FVS triangulations.

\section{References}

1. de Boor, C., R. A. DeVore, and A. Ron, On the construction of multivariate (pre) wavelets, Constr. Approx. 9 (1993), 123-166. 
2. de Boor, C., K. Höllig, and S. Riemenschneider, Box Splines, Springer, New York, 1993.

3. Buhmann, M. D. and C. A. Micchelli, Spline prewavelets on non-uniform knots, Numer. Math. 61 (1992), 455-474.

4. Carnicer, J. M. and J. M. Peña, Least supported bases and local linear independence, Numer. Math. 67 (1994), 289-301.

5. Ciarlet, P. G., Basic error estimates for elliptic problems, in Handbook of Numerical Analysis, Vol.II: Finite Element Methods (Part 1), P. G. Ciarlet and J. L. Lions (eds), North-Holland, Amsterdam, 1991, 17-351.

6. Chui, C. K., Multivariate Splines, CBMS-NSF Reg. Conf. Series in Appl. Math., vol. 54, SIAM, Philadelphia, 1988.

7. Chui, C. K., K. Jetter, and J. Stöckler, Wavelets and frames on the four-directional mesh, in Wavelets: Theory, Algorithms, and Applications, C. Chui, L. Montefusco, and L. Puccio (eds), Academic Press, New York, 1994, 213-230.

8. Conti, C. and K. Jetter, A new subdivision method for bivariate splines on the four-directional mesh, J. Comput. Appl. Math. 119 (2000), 81-96.

9. Dahmen, W., Wavelet and multiscale methods for operator equations, Acta Numerica 6 (1997), 55-228.

10. Dahmen, W. and C. A. Micchelli, Translates of multivariate splines, Linear Algebra Appl. 52 (1983), 217-234.

11. Dahmen, W., P. Oswald, and X.-Q. Shi, $C^{1}$-hierarchical bases, J. Comput. Appl. Math. 51 (1994), 37-56.

12. Dahmen, W. and R. Stevenson, Element-by-element construction of wavelets satisfying stability and moment conditions, SIAM J. Numer. Anal. 37 (1999), 319-352.

13. Daubechies, I., Ten Lectures on Wavelets, SIAM, Philadelphia, 1992.

14. Davydov, O., Stable local bases for multivariate spline spaces, J. Approx. Theory 111 (2001), 267-297.

15. Dæhlen, M., T. Lyche, K. Mørken, R. Schneider, and H.-P. Seidel, Multiresolution analysis over triangles, based on quadratic Hermite interpolation, J. Comput. Appl. Math. 119 (2000), 97-114.

16. DeVore, R. A. and B. Lucier, Wavelets, Acta Numerica 1 (1992), 1-56.

17. Floater, M. and E. Quak, Piecewise linear prewavelets on arbitrary triangulations, Numer. Math. 82 (1999), 221-252.

18. Fraeijs de Veubeke, G., Bending and stretching of plates, (Conference on Matrix Methods in Structural Mechanics), Wright-Patterson A.F.B. (OH), 1965, 863-886.

19. Lai, M.-J., Approximation order from bivariate $C^{1}$ cubics on a four-directional mesh is full, Comput. Aided Geom. Design 11 (1994), 215-223.

20. Lai, M.-J., Bivariate spline spaces on FVS-triangulations, in Approximation Theory VIII, Vol. 1: Approximation and Interpolation, Charles K. Chui and Larry L. Schumaker (eds), World Scientific Publishing Co., Inc., Singapore, 1995, 309-316. 
21. Lyche, T., K. Mørken, and E. Quak, Theory and algorithms for non-uniform spline wavelets, in Multivariate Approximation and Applications, N. Dyn, D. Leviatan, D. Levin, and A. Pinkus (eds), Cambridge University Press, 2001, 152-187.

22. Powell, M. J. D., Piecewise quadratic surface fitting for contour plotting, in Software for Numerical Mathematics, D. J. Evans (ed), Academic Press (London), 1974, 253271.

23. Powell, M. J. D. and M. A. Sabin, Piecewise quadratic approximations on triangles, ACM Trans. Math. Software 3 (1977), 316-325.

24. Ron, A. and Z. Shen, Compactly supported tight affine spline frames in $L^{2}\left(\mathbb{R}^{d}\right)$, Math. Comp. 67 (1998), 191-207.

25. Oswald, P., Hierarchical conforming finite element methods for the biharmonic equation, SIAM J. Numer. Anal. 29 (1992), 1610-1625.

26. Sander, G., Bornes supérieures et inférieures dans l'analyse matricielle des plaques en flexion-torsion, Bull. Soc. Royale Sciences Liège 33 (1964), 456-494.

27. Stevenson, R., Piecewise linear (pre-)wavelets on non-uniform meshes, in Multigrid Methods V, W. Hackbusch and G. Wittum (eds), Springer, Berlin, 1998, 306-319.

28. Zwart, P. B., Multivariate splines with non-degenerate partitions, SIAM J. Numer. Anal. 10 (1973), 665-673. 